Original Paper http://ajol.info/index.php/ijbcs http://indexmedicus.afro.who.int

\title{
Etat d'eutrophisation de la lagune continentale Ono (Bonoua sud-est de la Côte d'Ivoire) dans un environnement agro-industriel durant la crue du fleuve Comoé
}

\author{
Affoua Jeanne GBOKO ${ }^{1 *}$, Apie Colette $\mathrm{AKOBE}^{1}$, Ané Maurice AKA ${ }^{1}$, \\ Charles Albéric AKA ${ }^{1}$, Aka Ferdin KOUAME ${ }^{1}$, Koffi Nestor ADOU ${ }^{1}$, \\ Ossey Bernard $\mathrm{YAPO}^{2}$, Sylvain $\mathrm{MONDE}^{3}$ et Kouamé $\mathrm{AKA}^{3}$ \\ ${ }^{1}$ Département des Géosciences Marines, UFR STRM, Université Félix Houphouët-Boigny, Abidjan, \\ Côte d'Ivoire. \\ ${ }^{2}$ UFR des Sciences et gestions de l'environnement, Université Nangui Abrogoua, Abidjan, \\ Côte d'Ivoire. \\ ${ }^{3}$ Département des Géosciences marines, UFR STRM, Université Félix Houphouët-Boigny, Abidjan, \\ Côte d'Ivoire. \\ *Auteur correspondant ; E-mail: gbokoaffouajeanne@gmail.com
}

\section{RESUME}

Cette étude porte sur la variabilité spatiale et l'analyse statistique des paramètres d'eutrophisation obtenus sur onze (11) stations au niveau de la lagune Ono. Les données ont étés obtenues, à partir de mesures in-situ de paramètres (matière organique (MO), Oxygène dissous (OD), transparence) d'une part et d'autre part, de dosages de sels nutritifs par le spectromètre de masse DR 6000 du Centre Ivoirien Anti-pollution (CIAPOL). Il ressort de ces analyses que les teneurs en phosphate obtenues sont élevées avec une moyenne de $0,693 \mathrm{mg} / \mathrm{l}$. Elles sont à la base du bloom phytoplanctonique. Les teneurs faibles en oxygène dissous avec une moyenne de $1,879 \mathrm{mg} / \mathrm{l}$ sont dues à la forte concentration en matière organique (MO) des eaux en décomposition. Ce qui explique la corrélation inverse entre la MO et l'OD ( $\mathrm{r}=-0,52)$. La forte teneur en chlorophylle-a avec une moyenne de $5,763 \mu \mathrm{g} / \mathrm{l}$ explique la forte activité photosynthétique de cette lagune. Ces résultats obtenus montrent que les eaux de la lagune Ono sont eutrophes à oligotrophes en allant du Sud-Ouest au Nord-Est. Cette dégradation est occasionnée par les teneurs importantes de sels nutritifs surtout le phosphore. Ces sels sont issus du lessivage des terres agricoles et des rejets des eaux usées domestiques et industrielles du bassin versant. Ce qui induit la croissance accélérée des végétaux aquatiques terrestres et des algues. Ainsi, la décomposition de ces végétaux consomme l'oxygène dissous créant des conditions anoxiques. Le phénomène à l'origine de la pollution des eaux est par conséquent d'origine anthropique. Il faut donc mettre en place un programme de sensibilisation et de surveillance afin de réduire en amont l'apport des éléments nutritifs dans la lagune.

(C) 2019 International Formulae Group. All rights reserved

Mots clés : Lagune continentale, Ono, eutrophisation, crue, Comoé, Côte d'Ivoire. 


\title{
State of eutrophization of the ono continental lagoon (Bonoua south-east of Côte d'Ivoire) in an agro-industrial environment during the cruise of the Comoe river
}

\begin{abstract}
This study deals with the spatial variability and the statistical analysis of the eutrophication parameters obtained on eleven (11) sites at the Ono lagoon. The data were obtained, from in-situ measurements of parameters (organic matter (OM), dissolved oxygen (DO), transparency) on the one hand and on the other hand nutrient dosing by the mass spectrometer. DR 6000 Ivorian Center Anti-pollution (CIAPOL). These analyzes show that the phosphate levels obtained are high with an average of $0.693 \mathrm{mg} / \mathrm{l}$. They are at the base of the phytoplankton bloom. The low levels of dissolved oxygen with an average of $1.879 \mathrm{mg} / \mathrm{l}$ are due to the high concentration of organic matter $(\mathrm{OM})$ in decaying water. This explains the inverse correlation between the OM and the DO $(r=-0.52)$. The high content of chlorophyll-a with an average of $5.763 \mu \mathrm{g} / \mathrm{l}$ explains the strong photosynthetic activity of that lagoon. These results show that the waters of the Ono lagoon are eutrophic to oligotrophic from southwestern to northeastern. This degradation is caused by the high levels of nutrients, especially phosphorus. These salts are derived from the leaching of farmland and discharges of domestic and industrial wastewater from the watershed. This induces the accelerated growth of terrestrial aquatic plants and algae. Thus, the decomposition of these plants consumes dissolved oxygen creating anoxic conditions. The phenomenon at the origin of water pollution is therefore of anthropic origin.

Therefore, an awareness and monitoring program must be put in place to reduce the input of nutrients into the lagoon upstream.
\end{abstract}

(C) 2019 International Formulae Group. All rights reserved

Keywords: Ono, continental lagoon, eutrophication, flood, Comoé, Ivory Coast.

\section{INTRODUCTION}

Le réseau hydrographique en Côte d'Ivoire comporte un important système lagunaire comprenant les lagunes Ebrié, Grand Lahou et Aby. Et des lagunes continentales dont Ono qui fait l'objet de notre étude.

Cette lagune naturellement influencée par le fleuve Comoé, constitue un vaste plan d'eau du littoral Ivoirien. Elle est soumise à de fortes pressions anthropiques suscitées par le développement des activités agricoles, agroindustrielles et domestiques liées à la croissance démographique non contrôlée. Cette forte pression anthropique aboutit à une production de plus en plus importante de déchets de toutes natures sur les ressources naturelles en eau. Le rejet des sels nutritifs dans les eaux de surface favorise la croissance des végétaux aquatiques qui conduit à une pollution organique et des phénomènes d'eutrophisation (Ayah et al., 2015). Cette situation impacte la qualité des eaux et la surface de la lagune Ono. Elle est alors colonisée par les végétaux aquatiques et terrestres en entrainnant un rétrécissement graduel de sa surface. En effet, le bassin versant de la lagune Ono héberge des plantations d'une société de culture d'ananas. Les eaux de la lagune Ono sont alors destinées à irriguer les cultures de cette société en saison sèche. Une pêche traditionnelle s'y développe pour l'approvisionnement local en poissons. Malgré toutes ces activités économiques qui se réalisent dans son environnement, la lagune Ono semble être négligée par les recherches scientifiques. Les études réalisées sur la lagune Ono portent essentiellement sur les nombreuses espèces ichtyologiques (EYI et al., 2016) et sur les paramètres physico-chimiques de façon générale des eaux de surface de la région de Bonoua (Tohouri et al. (2017)). Cette problématique nous amène à étudier l'état d'eutrophisation de la lagune Ono durant la saison de crue du fleuve Comoé. Cette étude vise spécifiquement à maitriser la variabilité spatiale des paramètres d'eutrophisation et analyser l'état trophique des eaux de la lagune Ono.

\section{MATERIEL ET METHODES Matériel}

La lagune Ono est localisée au Nord de la ville de Bonoua à environ $49 \mathrm{Km}$. Elle a une superficie d'environ $6,1 \mathrm{~km}^{2}$ et est située 
entre les longitudes $3^{\circ} 35^{\prime} 20,62^{\prime}$ 'W $3^{\circ} 32^{\prime} 57,55^{\prime}$ ' W et les latitudes $5^{\circ} 21^{\prime} 10,44^{\prime}$ ' $\mathrm{N}$ - 5'23'34,30’' N. (Figure 1).

L'acquisition des données s'est faite sur onze (11) stations (Tableau 1). Au niveau de la surface et à l'interface eau-sédiment. Les paramètres tels que l'oxygène dissous et la transparence ont été mesurés «in situ ». Les teneurs en oxygène dissous ont été enregistrées à l'aide d'un multi paramètre $\mathrm{HO}$ 40d. La transparence de l'eau a été mesurée par le disque de Secchi. De l'eau a été prélevée dans des bocaux pour les analyses des sels nutritifs $\left(\mathrm{NH}_{4}{ }^{+}, \mathrm{NO}_{2}{ }^{-}, \mathrm{NO}_{3}{ }^{-}, \mathrm{PO}_{4}{ }^{3-}\right)$, de la matière organique et de la chlorophylle-a à l'aide du spectromètre de masse DR $6000 \mathrm{au}$ laboratoire.

\section{Acquisition des paramètres hydrologiques}

Pour la mesure de l'oxygène dissous, le multi-paramètre une fois connecté, est introduit directement dans l'eau, à chaque station. Les valeurs sont immédiatement affichées sur l'écran et sont ensuite relevées. Pour la transparence de l'eau, à l'aide d'une corde graduée attachée au disque, la profondeur à laquelle le disque de Secchi disparaît de la vue de l'observateur est la mesure notée. Les eaux prélevées ont été utilisées pour le dosage des sels nutritifs, la mesure de la teneur en matière organique et de la chlorophylle-a au laboratoire.

\section{Cartographie des paramètres hydrologiques}

Le logiciel ArcGIS a servi à la réalisation des cartes de répartition spatiale des différents paramètres hydrologiques. Elle peut se résumer en deux (2) phases dont la première consiste à élaborer avec le tableur Excel, un fichier CVS de données numériques des différents paramètres hydrologiques pour chaque station avec les données prélevées sur le terrain. A ce fichier on ajoute la carte de contour obtenue par digitalisation de la base de données cartographique du système lagunaire Ivoirien. La seconde phase est de sortir les cartes de répartition spatiale des différents paramètres en faisant une interpolation. La méthode d'interpolation utilisée est le krigeage.

\section{Analyse en composante principale}

Le nombre de variables étudiées et l'effectif des échantillons imposent un recours aux analyses multidimensionnelles. Rappelons que la matrice de corrélation et le cercle de corrélation des différents paramètres hydrologiques ont été élaborés au moyen du logiciel Statistica 7.1. L'analyse en Composantes Principales (ACP) permet de mettre en évidence les similitudes chimiques et la position graphique que représenteraient deux ou plusieurs variables chimiques au cours de leur évolution. Ainsi deux variables voisines sur le cercle de communauté ont une affinité. Cette analyse a permis d'avoir des informations pour l'interprétation hydrologique. $\mathrm{Ce}$ sont les paramètres statistiques et la matrice de corrélation entre les variables. Pour la présente étude, seule la matrice de corrélation sera utilisée pour apprécier l'affinité entre les différents paramètres étudiés.

Tableau 1: Coordonnées géographiques (UTM) et profondeurs (m) des stations de mesure.

\begin{tabular}{llllllllllll}
\hline & St 1 & St 2 & St 3 & St 4 & St 5 & St 6 & St 7 & St 8 & St 9 & St 10 & St 11 \\
\hline Long & 438654 & 437347 & 437466 & 437586 & 436498 & 436202 & 435351 & 435590 & 435111 & 434942 & 434772 \\
\hline Lat & 594988 & 594319 & 594010 & 593641 & 593222 & 593697 & 592892 & 592583 & 592293 & 592463 & 591804 \\
\hline $\begin{array}{l}\text { Prof } \\
(m)\end{array}$ & 1 & 1,905 & 2,037 & 2,071 & 2,152 & 2 & 1,861 & 2,142 & 2,491 & 2,473 & 3,748 \\
\hline
\end{tabular}

Long : Longitude, Lat : Latitude, Prof : Profondeur, St : station 


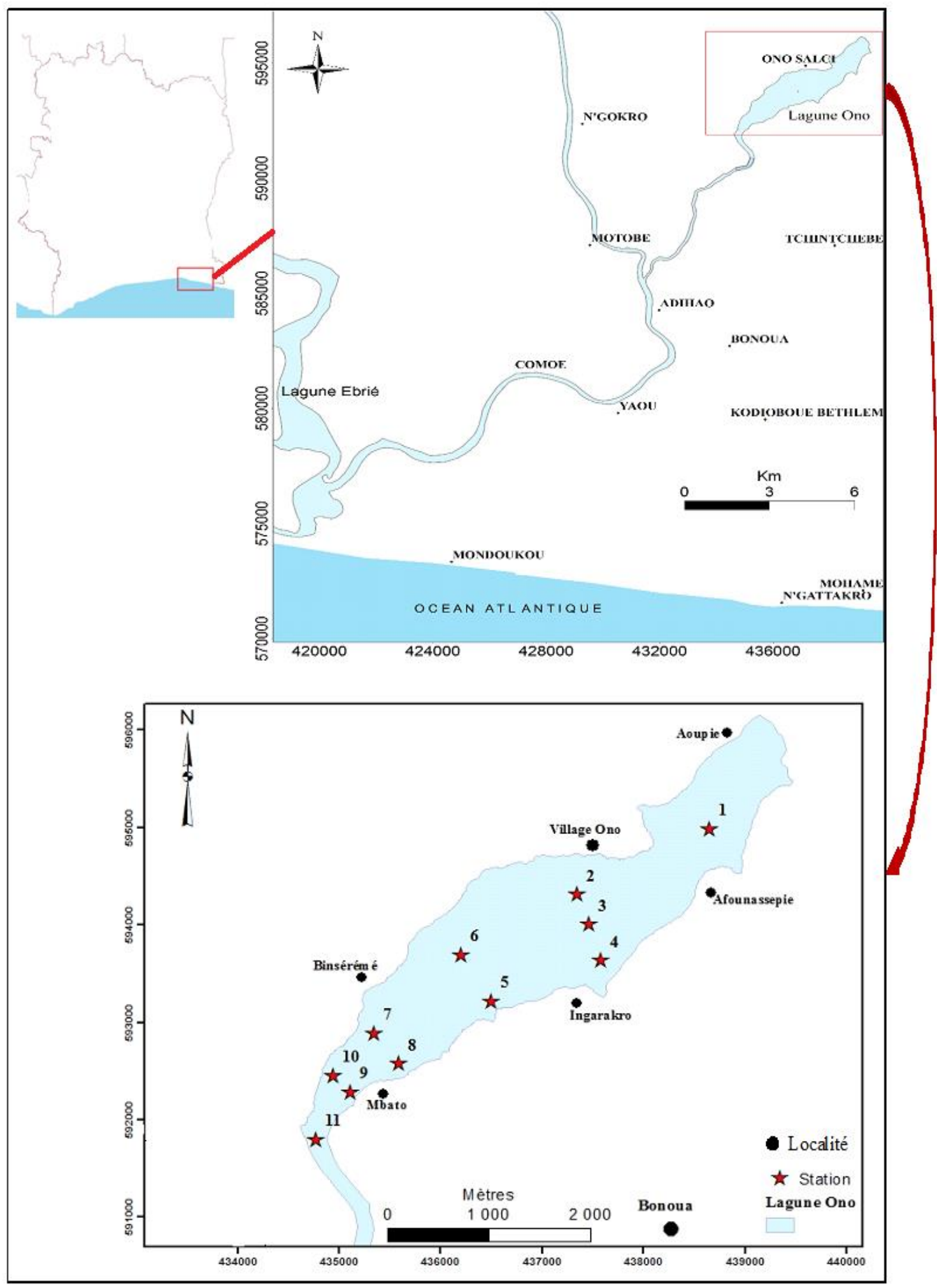

Figure 1 : Situation géographique et positionnement des points d'échantillonnages. 


\section{RESULTATS}

Les résultats d'analyse des paramètres d'eutrophisation sont consignés dans le Tableau 2. Ces valeurs enregistrées montrent les différentes variations au plan spatial et dans la colonne d'eau.

\section{Variation spatiale de la matière organique dans la lagune Ono}

La forte concentration de la matière organique est observée à l'extrémité SudOuest. Cette concentration diminue progressivement vers le Nord-Est (Figure 2).

Variation de la matière organique dans la colonne d'eau

Les teneurs en matière organique du fond sont plus élevées que celles de la surface (Figure 3).

\section{Variation spatiale de l'oxygène dissous dans la lagune Ono}

Les teneurs en oxygène dissous (OD) croissent du Centre vers les deux extrémités Nord et Sud (Figure 4). Les faibles teneurs sont observées au Sud-Ouest et au Nord-Est.

Teneur en oxygène dissous dans la colonne d'eau

Les valeurs de l'OD sont plus élevées en surface qu'en profondeur en tous points de mesure (Figure 5).

\section{Evolution spatiale de la transparence dans la lagune Ono}

La répartition de la transparence dans la lagune Ono n'est pas homogène (Figure 6). Sur cette carte, nous constatons que les eaux très chargées en matières en suspension se trouvent dans le Centre-Ouest et celles moyennement chargées dans les deux extremités. Au Centre-Est la penétration de la lumière dans la colonne d'eau est relativement considerable. Les eaux sont plus transparentes entre les villages Ono et Ingarakro.

\section{Variation spatiale de l'azote ammoniacal dans la lagune Ono}

Les fortes concentrations de l'ammonium sont observées dans la partie
Sud-Ouest au contact du fleuve Comoé (Figure 7).

Teneur de l'azote ammoniacal dans la colonne d'eau

De façon générale, les valeurs de l'ammonium dans la colonne d'eau sont plus élevées en profondeur qu'en surface. Sauf au niveau des stations 2,4 et 9 où les valeurs sont légèrement élevées en surface (Figure 8).

\section{Variation spatiale des nitrites dans la lagune Ono}

La répartition des nitrites dans la lagune Ono n'est pas homogène. Les fortes concentrations sont observées au Sud de la lagune (Figure 9). Les faibles valeurs sont enregistrées aux extrémités Ouest et Nord-Est.

\section{Teneur des nitrites dans la colonne d'eau}

Les nitrites ont des valeurs plus élevées au fond qu'en surface (Figure 10). Sauf au niveau des points $4,5,6,7,9$ où les teneurs en nitrite sont plus élevées en surface qu'au fond.

Variation spatiale des nitrates dans la lagune Ono

La répartition des nitrates dans les eaux de la lagune Ono est hétérogène. Les teneurs moyennes couvrent la quasi-totalité de la lagune, les fortes teneurs sont en bleu et les faibles en rouge bordé de jaune (Figure 11).

\section{Teneur des nitrates dans la colonne d'eau}

Le nitrate a des valeurs plus élevées en surface qu'au fond. Sauf au niveau des points $6,7,8$ où les valeurs sont plus élevées en surface qu'au fond (Figure 12).

\section{Variation de l'orthophosphate dans la lagune Ono}

Les zones de teneurs élevées en orthophosphates matérialisées par la couleur bleue sont observées au niveau de la bordure Nord. Les faibles teneurs matérialisées par la couleur marron sont observées au niveau de la bordure Sud (Figure 13).

Teneur de l'orthophosphate dans la colonne d'eau

Les teneurs de l'orthophosphate sont plus élevées au fond qu'en surface sauf aux 
stations 9 et 11 (Figure 14). La zone Sud concentre les plus faibles valeurs.

\section{Variation de la chlorophylle-a dans la lagune Ono}

Les fortes concentrations de la chlorophylle-a sont observées dans la partie Nord-Ouest de la lagune et décroit progressivement vers le Sud et le Nord-Est (Figure 15).

\section{Degrés de corrélation des paramètres d'eutrophisation}

Des corrélations positives et inverses sont observées dans le Tableau 3.

Une corrélation moyenne $(\mathrm{r}=0,54)$ existe entre $\mathrm{NO}_{3}{ }^{-}$et $\mathrm{NO}_{2}{ }^{-}$, aussi entre le OD et le $\mathrm{NO}_{2}{ }^{-}(\mathrm{r}=0,50)$. La MO et le OD sont inversement corrélés $(r=-0,52)$. Il existe une bonne corrélation $(\mathrm{r}=0,76)$ entre la chlorophylle-a et la MO.

Tableau 2 : Teneurs des paramètres d'eutrophisation.

\begin{tabular}{|c|c|c|c|c|c|c|c|c|c|c|c|c|c|c|}
\hline Paramètres & Prof & St 1 & St 2 & St 3 & St 4 & St 5 & St 6 & St 7 & St 8 & St 9 & St 10 & St 11 & Moy & E.type \\
\hline \multirow{2}{*}{$\begin{array}{l}\text { Matière organique } \\
\text { (mg/l) }\end{array}$} & $S$ & 4 & 4 & 3 & 4 & 5 & 4 & 6 & 5 & 8 & 8 & 8 & 5,36 & 1,86 \\
\hline & $F$ & 31 & 7,8 & 25,6 & 7 & 18 & 21 & 6 & 5 & 7,6 & 8,6 & 10,6 & 13,47 & 8,94 \\
\hline \multirow{2}{*}{$\begin{array}{l}\text { Oxygène dissous } \\
\text { (mg/l) }\end{array}$} & $S$ & 2,23 & 1,91 & 3,49 & 4,74 & 3,57 & 5,84 & 2,81 & 2,39 & 1,92 & 2,4 & 1,73 & 3,003 & 1,30 \\
\hline & $F$ & 1,39 & 0,26 & 2,21 & 1,28 & 1,09 & 0,42 & 0,18 & 0,81 & 0,34 & 0,2 & 0,12 & 0,755 & 0,67 \\
\hline
\end{tabular}

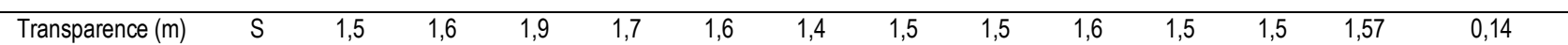

\begin{tabular}{|c|c|c|c|c|c|c|c|c|c|c|c|c|c|c|}
\hline \multirow{2}{*}{$\begin{array}{l}\text { Ammo } \\
\text { nium }(\mathrm{mg} / \mathrm{l})\end{array}$} & $S$ & 0,01 & 0,05 & 0 & 0,04 & 0,03 & 0,01 & 0,01 & 0 & 0,02 & 0,01 & 0,03 & 0,019 & 0,016 \\
\hline & $F$ & 0,03 & 0 & 0,01 & 0,02 & 0,05 & 0,02 & 0,03 & 0,02 & 0,01 & 0,11 & 0,23 & 0,05 & 0,07 \\
\hline \multirow[t]{2}{*}{ Nitrite $(\mathrm{mg} / \mathrm{l})$} & $S$ & 0,003 & 0,007 & 0,009 & 0,014 & 0,022 & 0,014 & 0,008 & 0,009 & 0,013 & 0,001 & 0,01 & 0,01 & 0,006 \\
\hline & $\bar{F}$ & 0,014 & 0,011 & 0,012 & 0,007 & 0,02 & 0,005 & $\overline{0,007}$ & $\overline{0,021}$ & $\overline{0,007}$ & $\overline{0,006}$ & 0,006 & 0,011 & 0,006 \\
\hline
\end{tabular}

\begin{tabular}{|c|c|c|c|c|c|c|c|c|c|c|c|c|c|c|}
\hline \multirow[t]{2}{*}{ Nitrate $(\mathrm{mg} / \mathrm{l})$} & $\mathrm{S}$ & 1,1 & 0,9 & 1,3 & 1,2 & 1,2 & 1,4 & 1 & 0,6 & 1,7 & 0,5 & 0,8 & 1,06 & 0,35 \\
\hline & $\mathrm{F}$ & 1,1 & 0,1 & 0,8 & 0,1 & 0,9 & 2 & 2,5 & 1,3 & 0,5 & 0,4 & 0,1 & 0,89 & 0,79 \\
\hline \multirow{2}{*}{$\begin{array}{l}\text { Ortho- } \\
\text { phosphate (mg/l) }\end{array}$} & S & 0,62 & 0,75 & 0,66 & 0,6 & 0,48 & 0,69 & 0,71 & 0,57 & 0,66 & 0,6 & 0,66 & 0,64 & 0,07 \\
\hline & $\mathrm{F}$ & 0,81 & 0,88 & 0,7 & 0,73 & 0,63 & 0,91 & 0,91 & 0,63 & 0,57 & 1 & 0,49 & 0,75 & 0,16 \\
\hline
\end{tabular}

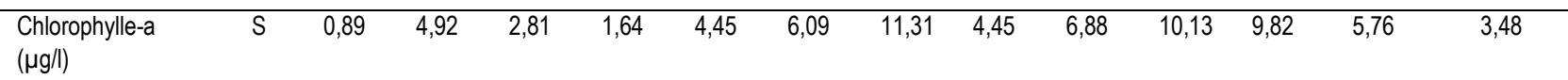


A. J. GBOKO et al. / Int. J. Biol. Chem. Sci. 13(6): 2942-2958, 2019

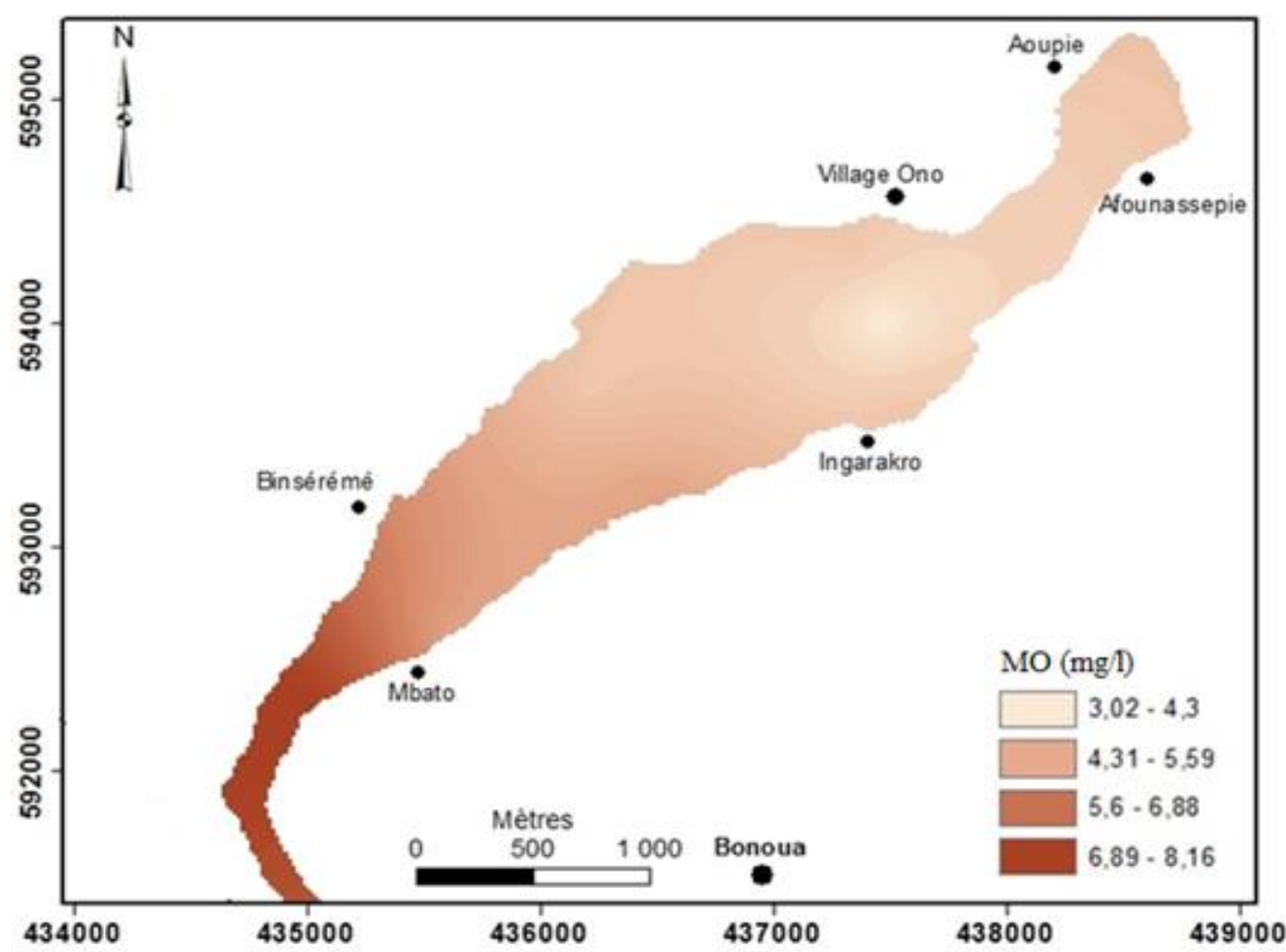

Figure 2 : Carte de répartition de la matière organique des eaux superficielles.

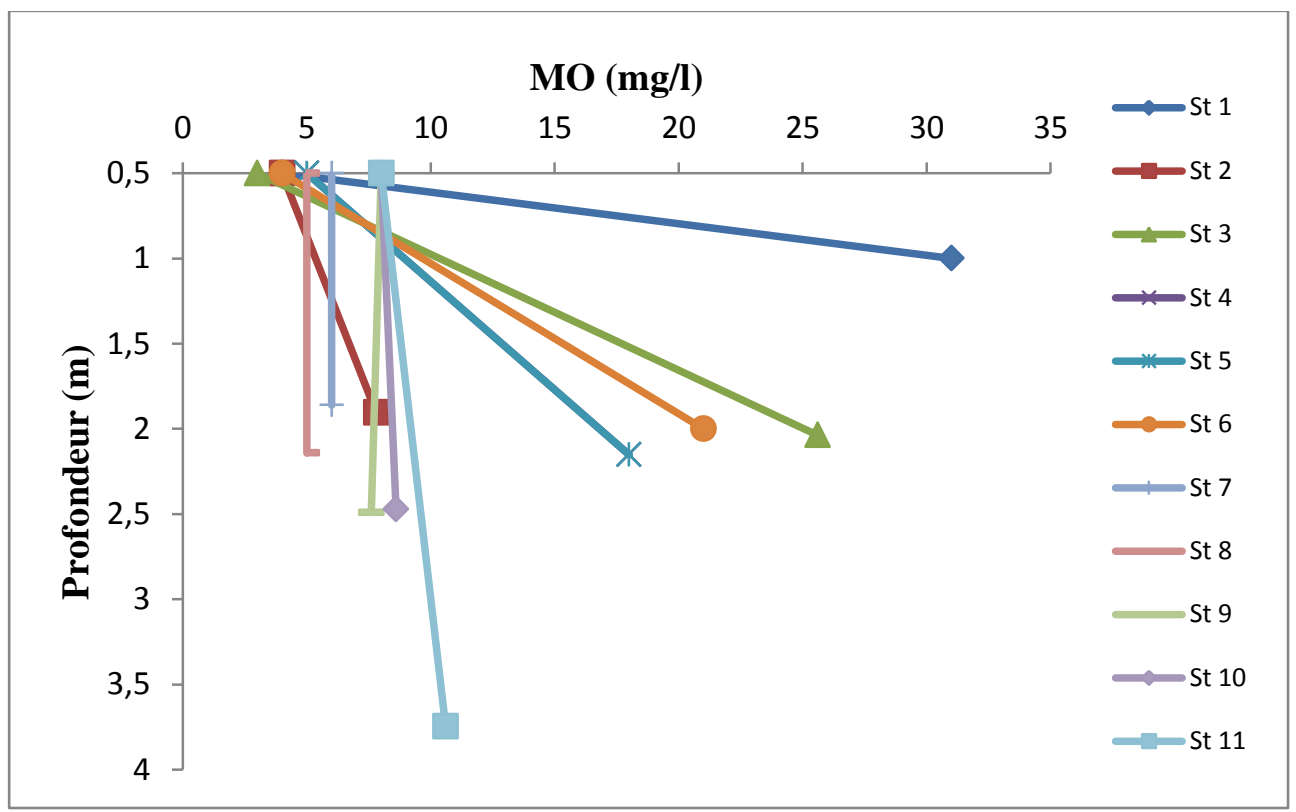

Figure 3 : Variation de la teneur en matière organique en fonction de la profondeur. 
A. J. GBOKO et al. / Int. J. Biol. Chem. Sci. 13(6): 2942-2958, 2019

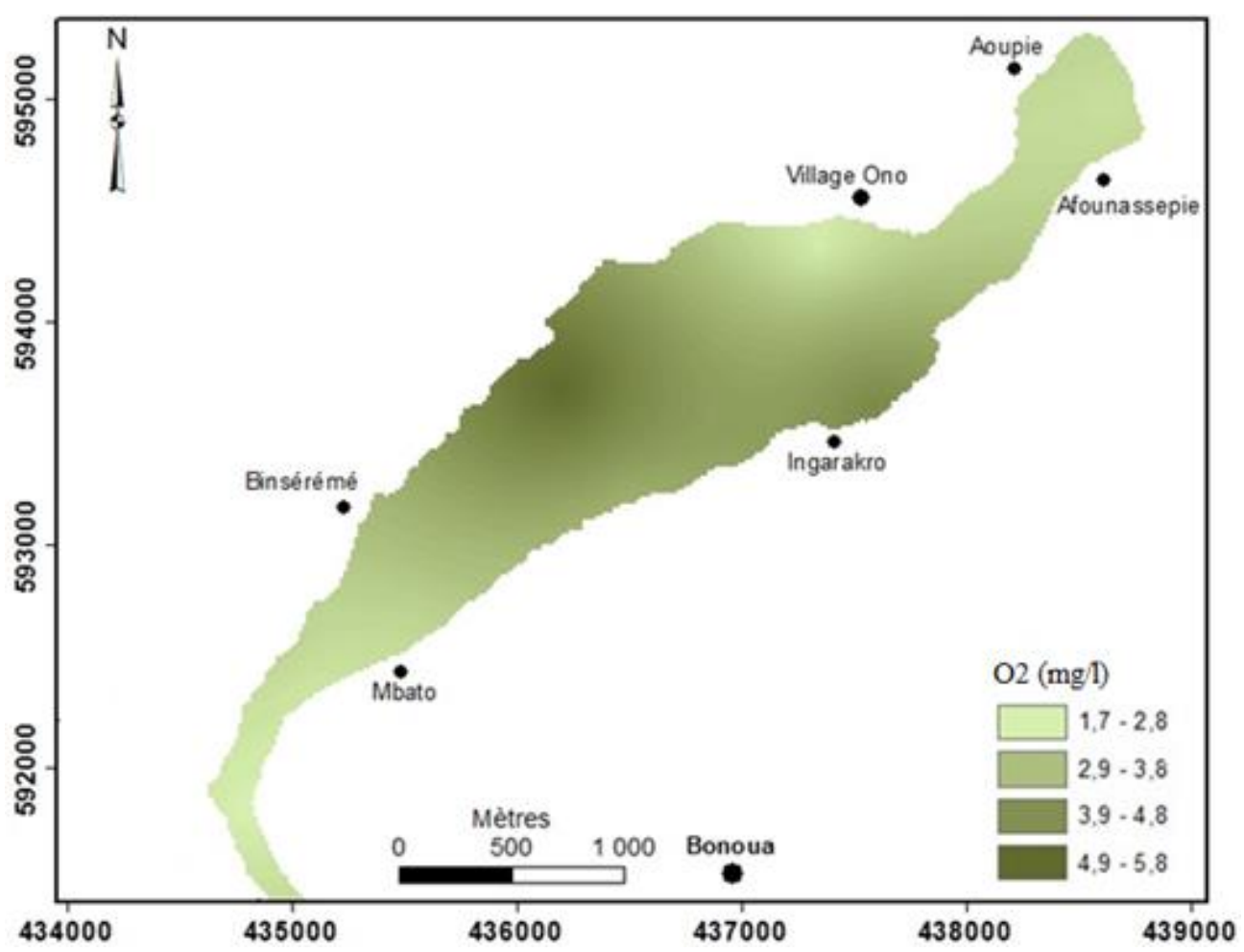

Figure 4 : Carte de répartition de la teneur en oxygène dissous des eaux superficielles.

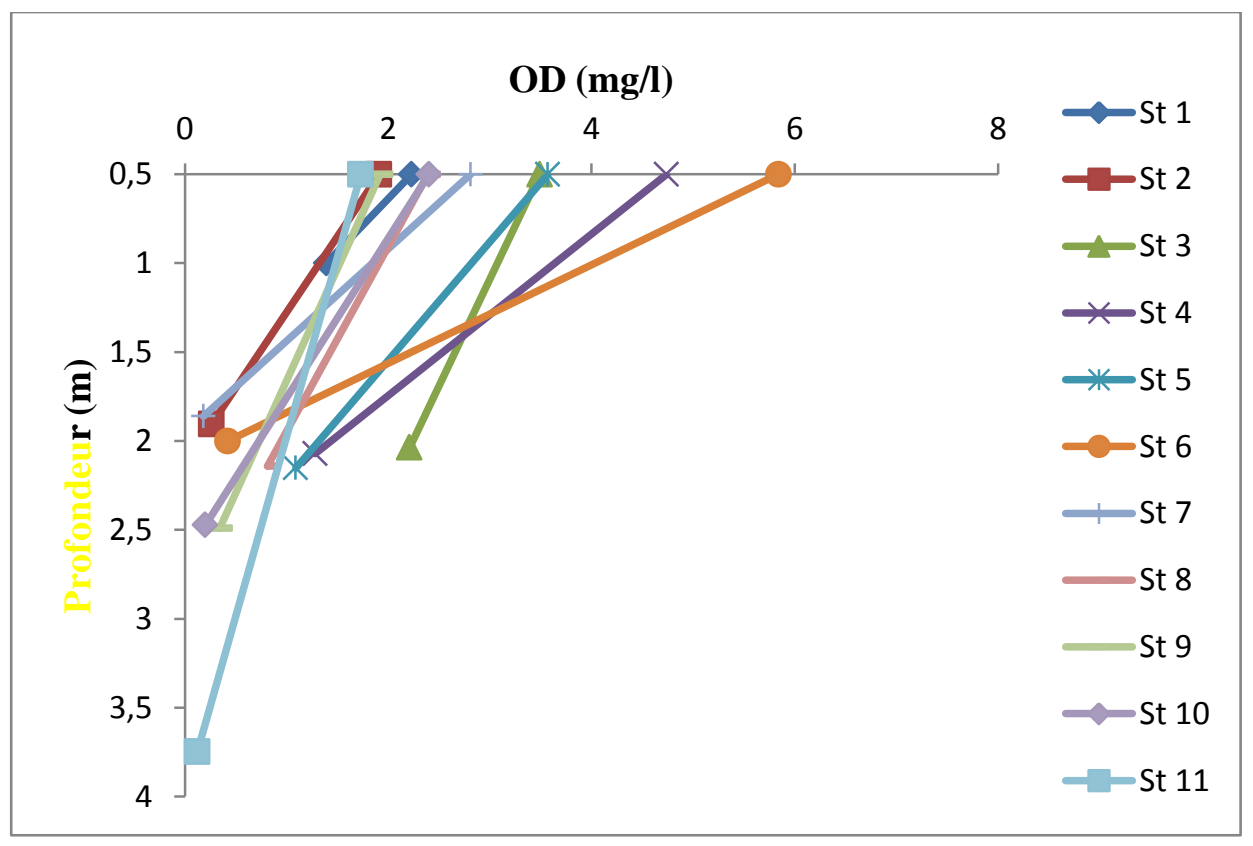

Figure 5 : Variation de la teneur en oxygène dissous suivant la profondeur. 


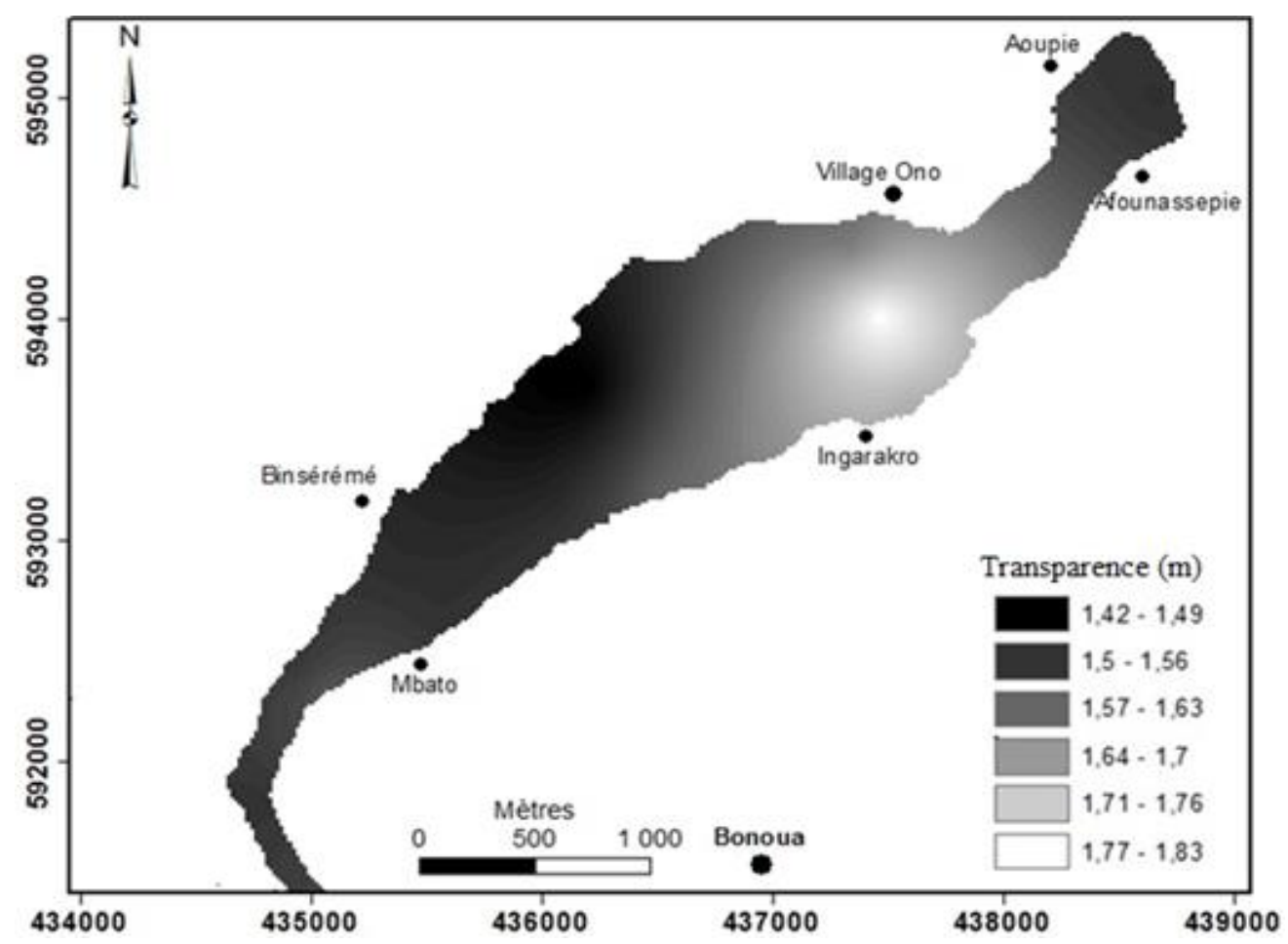

Figure 6 : Carte de répartition de la transparence.

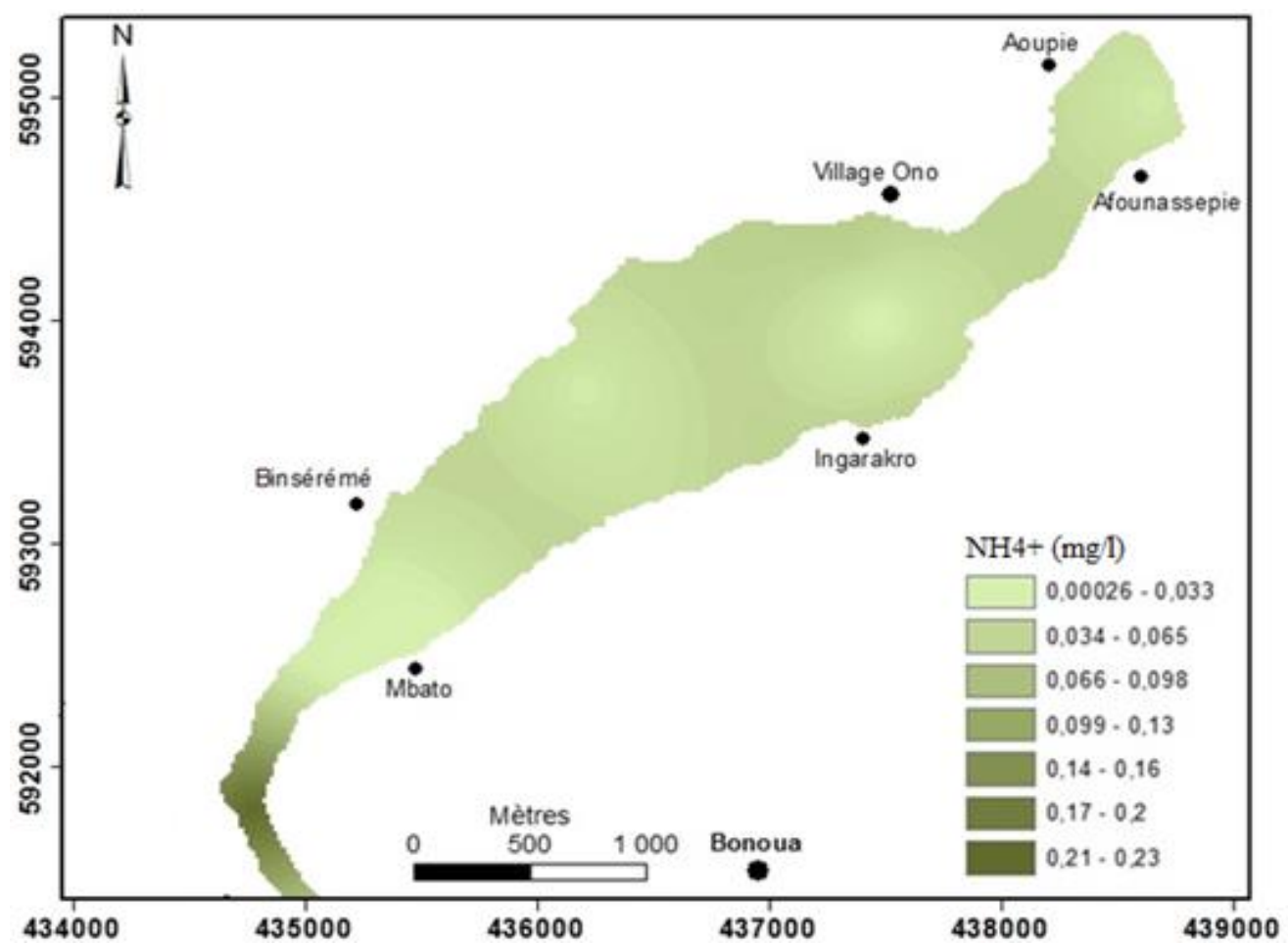

Figure 7 : Carte de répartition de l'azote ammoniacal $\left(\mathrm{NH}_{4}{ }^{+}\right)$des eaux superficielles. 


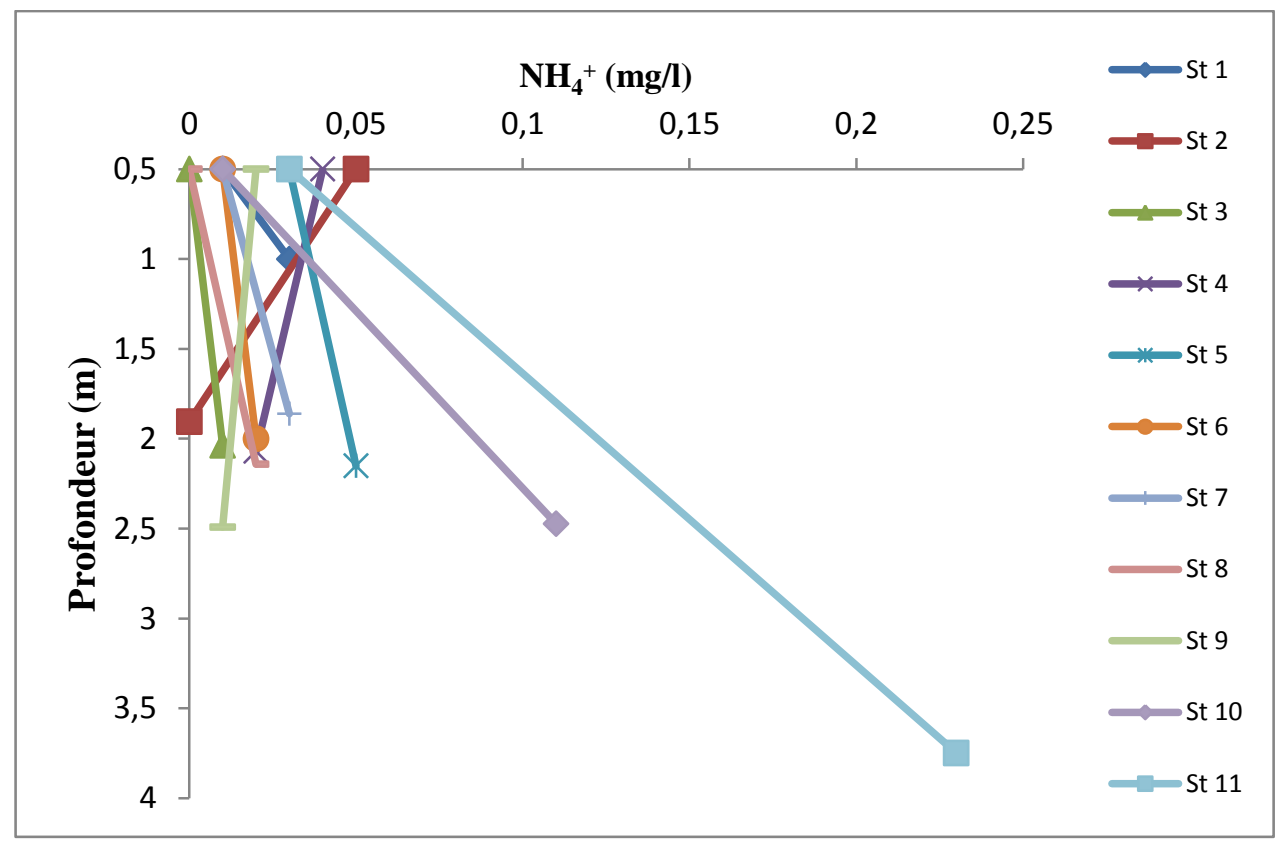

Figure 8 : Variation de la teneur en ammoniums suivant la profondeur.

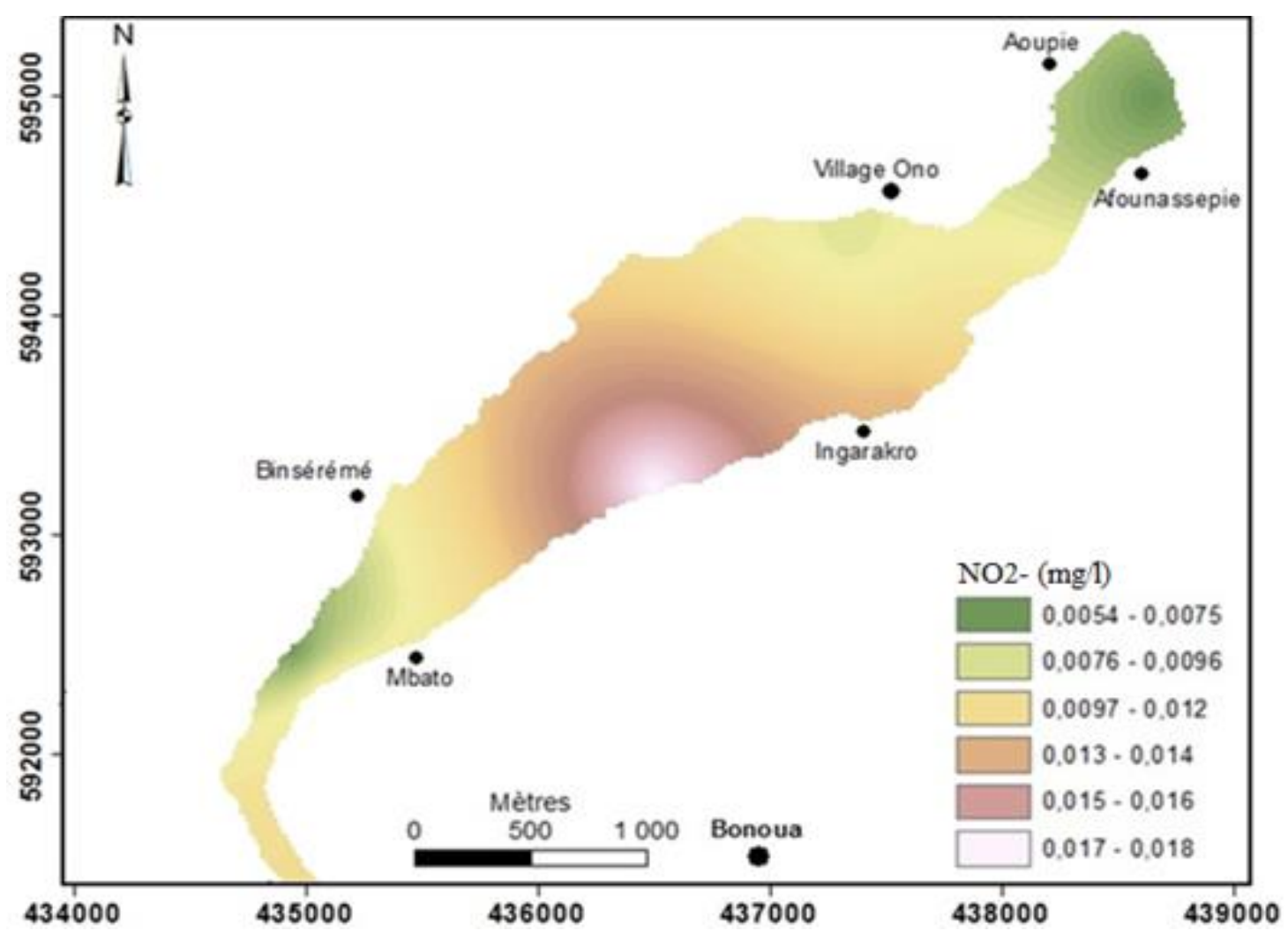

Figure 9 : Carte de répartition des nitrites $\left(\mathrm{NO}_{2}^{-}\right)$des eaux superficielles. 


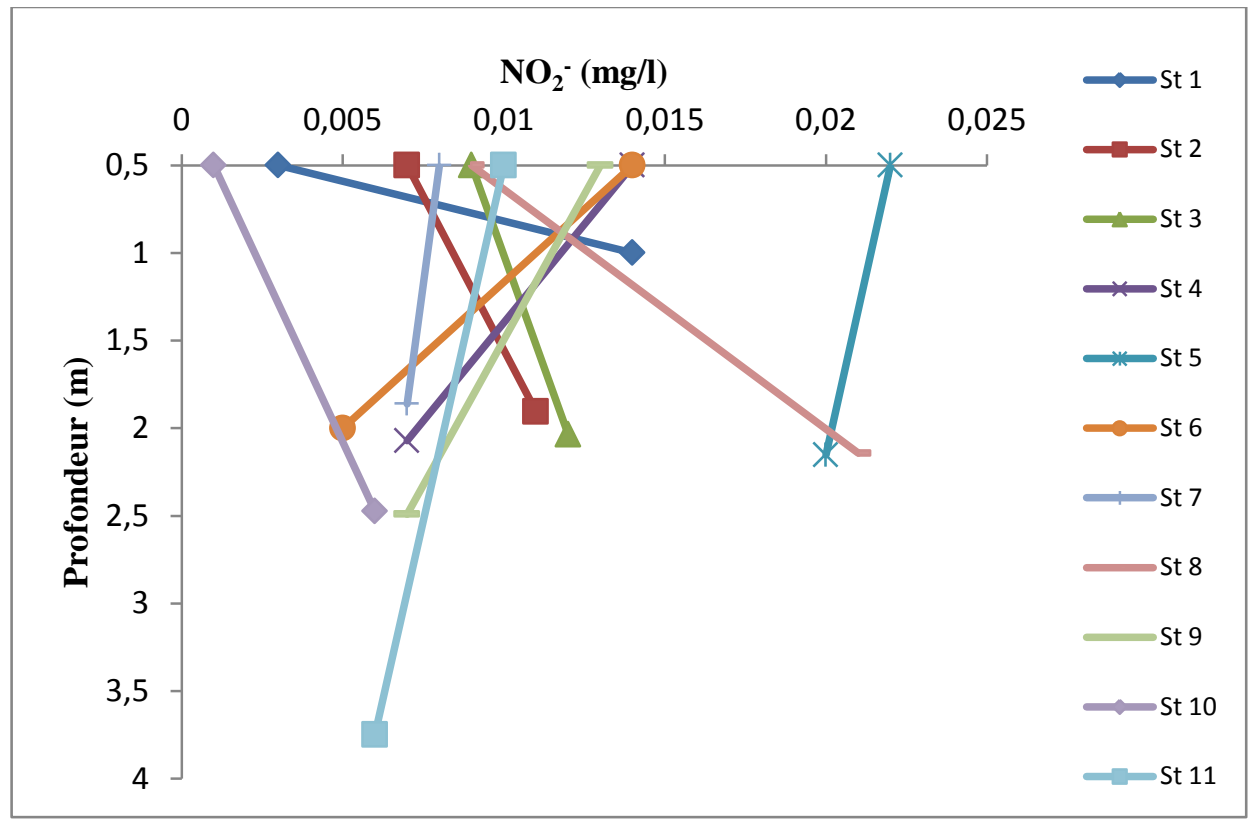

Figure 10 : Variation de la teneur en nitrites en fonction de la profondeur.

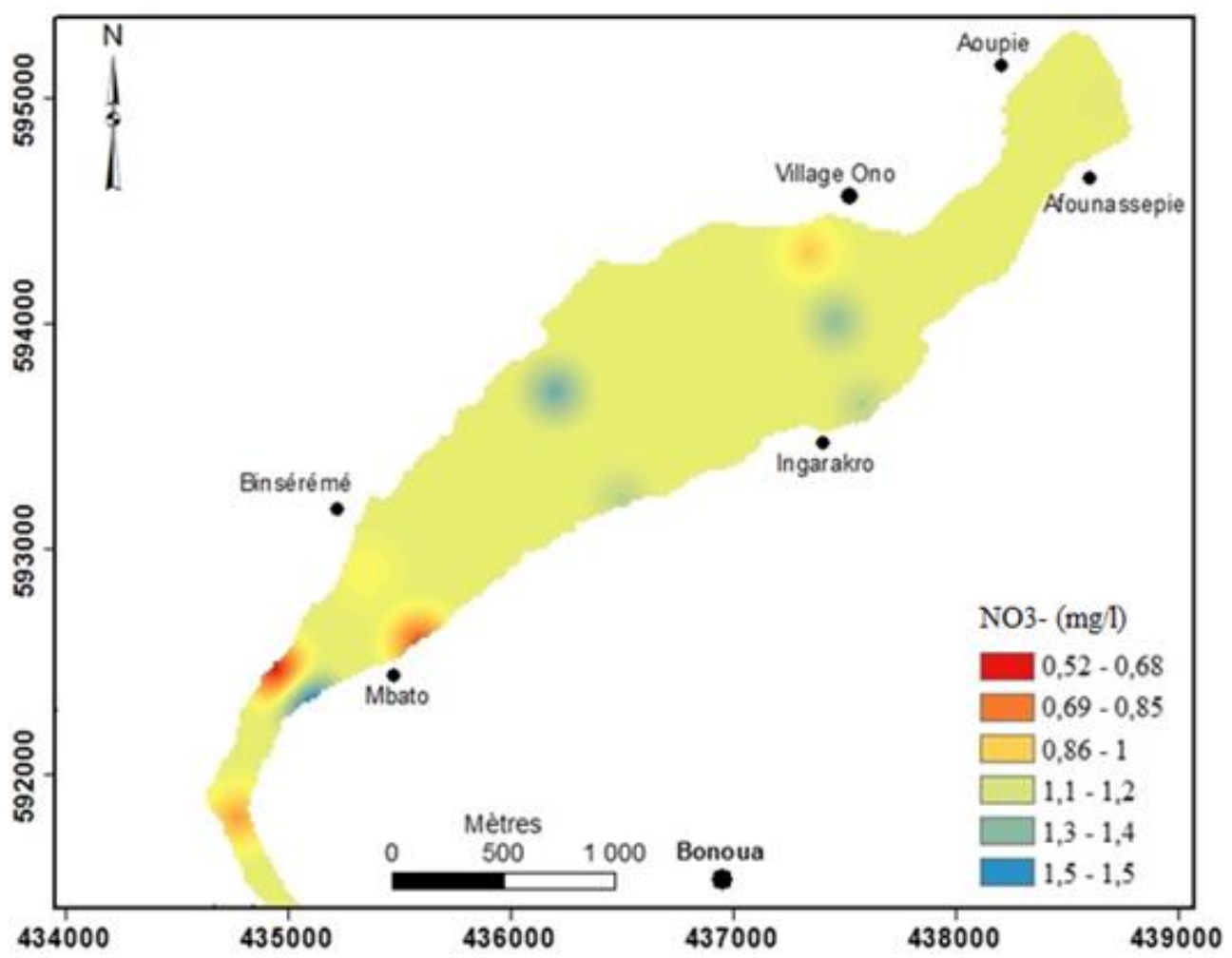

Figure 11 : Carte de répartition des nitrates $\left(\mathrm{NO}_{3}{ }^{-}\right)$des eaux superficielles 


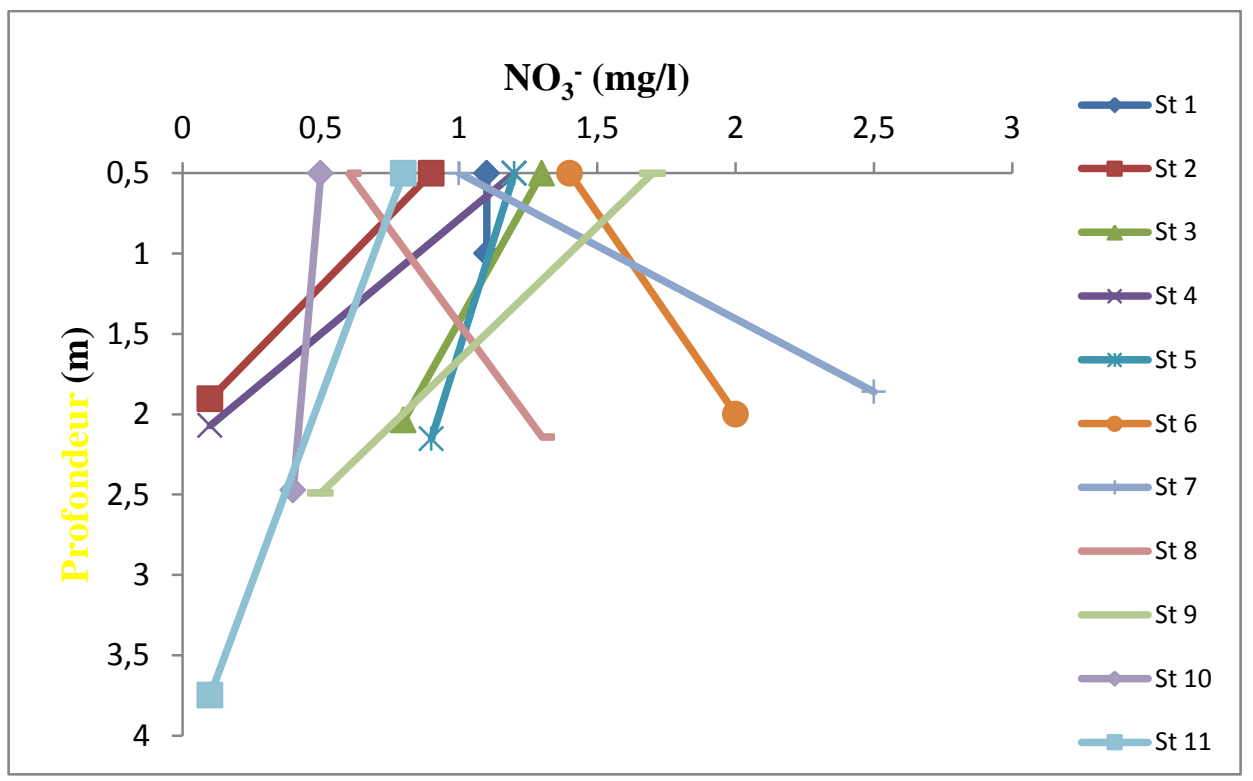

Figure 12 : Variation de la teneur en nitrates suivant la profondeur.

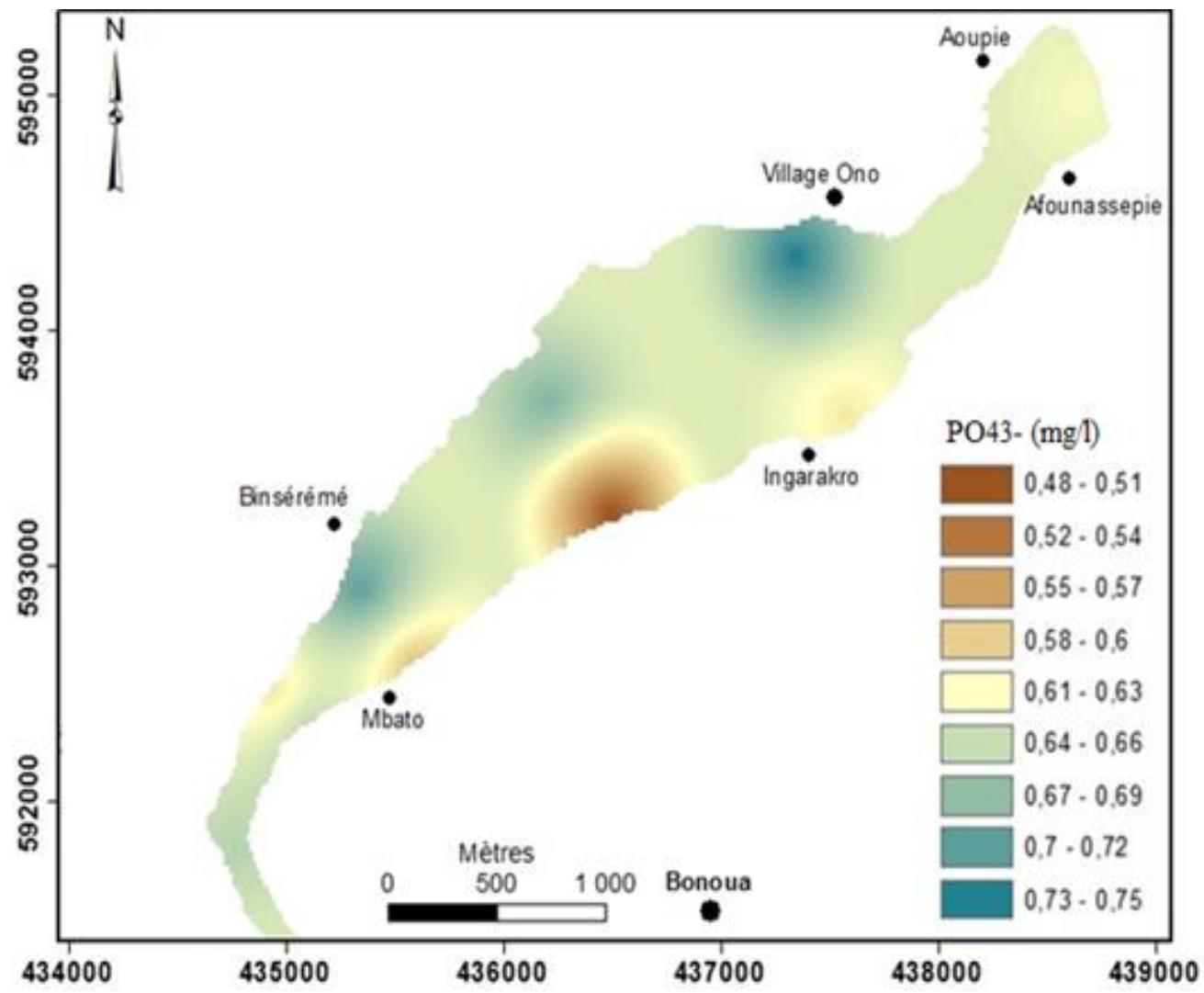

Figure 13 : Carte de répartition des orthophosphates $\left(\mathrm{PO}_{4}{ }^{3-}\right)$ des eaux superficielles. 


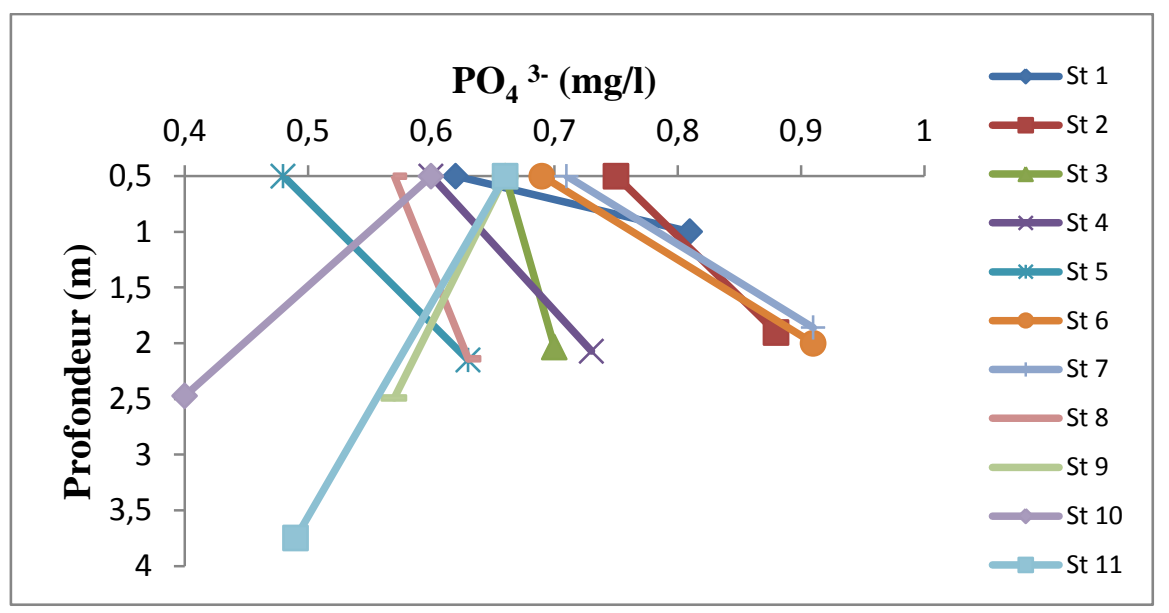

Figure 14 : Variation de la teneur en orthophosphates dans la colonne d'eau.

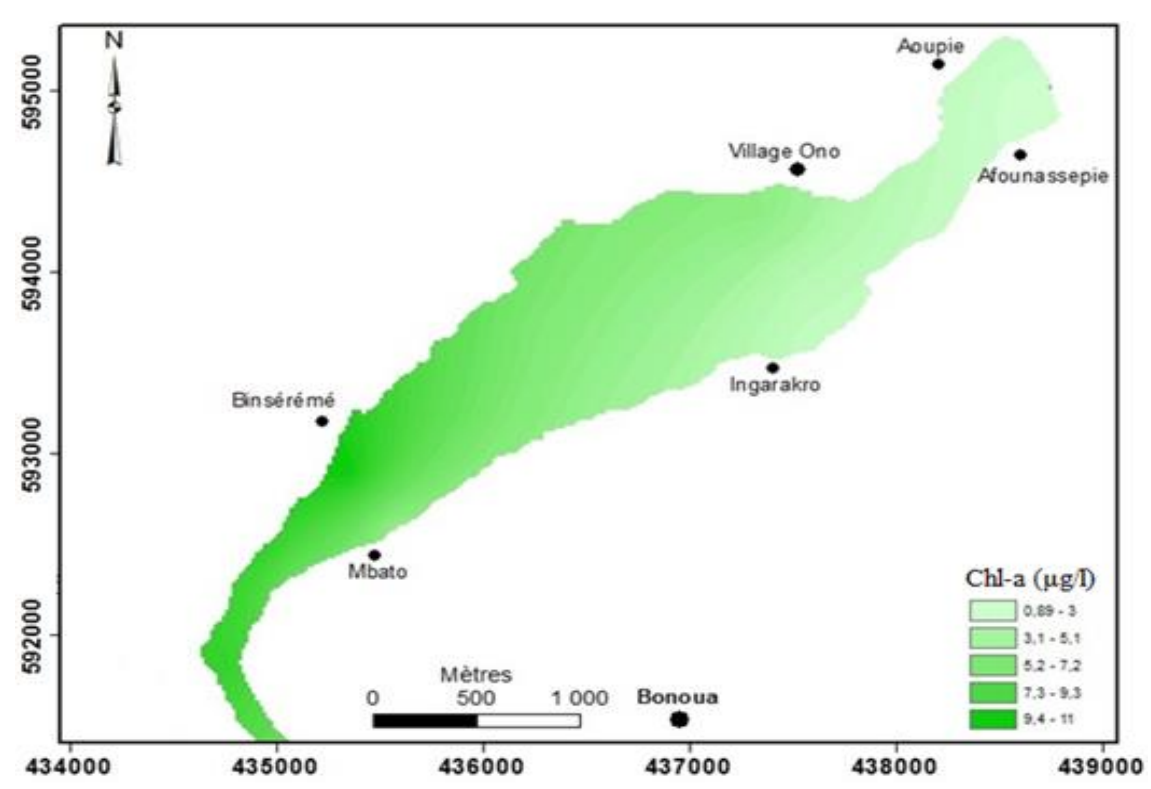

Figure 15 : Carte de répartition de la chlorophylle-a des eaux de la lagune Ono.

Tableau 3: Matrice de corrélation des paramètres d'eutrophisation

\begin{tabular}{cccccccc}
\hline Matrice & $\mathbf{P O}_{4}{ }^{3-}$ & $\mathbf{N O}_{2}{ }^{-}$ & $\mathbf{N O}_{3}{ }^{-}$ & $\mathbf{N H}_{4}{ }^{+}$ & $\mathbf{O D}$ & $\mathbf{M O}$ & $\mathbf{C h l - a}$ \\
\hline $\mathbf{P O}_{4}{ }^{3-}$ & 1 & & & & & & \\
$\mathbf{N O}_{\mathbf{2}}{ }^{-}$ & $-0,40$ & 1 & & & & & \\
$\mathbf{N O}_{3}{ }^{-}$ & 0,12 & 0,54 & 1 & & & & \\
$\mathbf{N H}_{\mathbf{4}}{ }^{-}$ & 0,12 & 0,07 & $-0,21$ & 1 & & & \\
$\mathbf{O D}$ & $-0,11$ & 0,50 & 0,39 & $-0,31$ & 1 & & \\
$\mathbf{M O}$ & $-0,04$ & $-0,14$ & $-0,22$ & 0,43 & $-0,52$ & 1 & \\
$\mathbf{C h l - a}$ & 0,28 & $-0,18$ & $-0,32$ & 0,33 & $-0,28$ & 0,76 & 1 \\
\hline
\end{tabular}




\section{DISCUSSION}

La teneur moyenne de l'oxygène dissous obtenue dans la lagune Ono est de $1,879 \mathrm{mg} / \mathrm{l}$. La teneur était plus faible avec une moyenne de 1,08 mg/l (Eyi et al., 2016). Selon l'IBGE, (2005), fixant la teneur moyenne des eaux de surface à $5 \mathrm{mg} / \mathrm{l}$, cette eau est fortement chargée en éléments organiques.

Ces faibles teneurs sont dues à l'abondance de la matière organique en décomposition dans la lagune Ono malgré la forte productivité photosynthétique des végétaux aquatiques et terrestres. $\mathrm{La}$ corrélation inverse $(r=-0,52)$ entre la MO et le OD dans l'analyse statistique présente clairement leur évolution en sens contraire. En effet, la demande en oxygène pour l'autoépuration de la lagune est élevée vu sa forte concentration en matières organiques. Dans les couches plus profondes rendues obscures n'ont lieu que des phénomènes consommateurs d'oxygène (respirations et dégradations). L'oxygène y devient limitant. $\mathrm{Ce}$ qui est favorable à tout processus d'eutrophisation. Ce phénomène occupe près de $2 / 5$ de la lagune avec la présence de végétaux aquatiques et terrestres envahissants. Dans l'extrémité Sud-Ouest, influencée par les rejets d'eau du fleuve Comoé, le courant catalyse l'oxydation de la MO. La teneur en $\mathrm{OD}$ en cette zone est inférieure à $1 \mathrm{mg} / \mathrm{l}$ indiquant un état proche de l'anaérobiose selon les normes de l'IBGE, (2005). Cet environnement lagunaire présente une crise dystrophique, plus ou moins réversible par endroit surtout dans le fond déséquilibrant cet écosystème (SEEE, 2008). Ces eaux sont faiblement oxygénées par rapport à celles de la lagune Aghien-Potou avec une moyenne de $4,9 \mathrm{mg} / \mathrm{l}$ (Traoré et al., 2012). La forte concentration de la matière organique observée à l'extrémité Sud-Ouest est due à l'écoulement du fleuve Comoé vers cet endroit ne favorisant pas la décantation de celle-ci.

Les teneurs élevées de l'OD enregistrées au niveau des extrémités Nord et
Sud auraient un lien avec la faible concentration en matière organique. En effet, dans certains écosystèmes, la production « in situ » par la photosynthèse peut engendrer des sursaturations atteignant 150 voire plus de 200 $\%$. Mais l'accumulation de matières organiques peut conduire à la consommation de l'oxygène dissous par les bactéries hétérotrophes et induire des sous-saturations voire l'anoxie (Ifremer, 2001).

Dans la colonne d'eau, les faibles teneurs de l'OD observées au fond s'expliqueraient par la décomposition de la matière organique accumulée au fond qu'en surface. Quant à la faible transparence mesurée avec une moyenne de $1,57 \mathrm{~m}$ témoignerait d'une forte turbidité de l'eau au moment des mesures.

Les sels nutritifs (ammoniums $\left(\mathrm{NH}_{4}{ }^{+}\right)$ nitrites $\left(\mathrm{NO}_{2}^{-}\right)$, nitrates $\left(\mathrm{NO}_{3}^{-}\right)$et orthophosphates $\left(\mathrm{PO}_{4}{ }^{3-}\right)$ ) sont des paramètres importants pour le suivi de la qualité des eaux de surface. Selon l'IBGE (2005), ils constituent les matières nutritives de choix pour les végétaux aquatiques et terrestres. Les normes établies par l'IBGE, (2005) sont de 50 $\mathrm{mg} \mathrm{NO}_{3}{ }^{-} / 1,3 \mathrm{mg} \mathrm{NO}_{2}{ }^{-} / 1$ et $3 \mathrm{mg} \mathrm{NH}_{4}{ }^{+} / 1$ et 0,15 $\mathrm{mg} / \mathrm{l}$ pour les orthophosphates.

La variation des teneurs en sels azotés (ammonium, les nitrites, et les nitrates) résulte de la forte activité bactérienne dégradant la $\mathrm{MO}$ en $\mathrm{NH}_{4}{ }^{+}$puis, par oxydation en $\mathrm{NO}_{2}{ }^{-}$et $\mathrm{NO}_{3}^{-}$. Ils sont issus des rejets d'eaux usées domestiques et du lessivage des terres agricoles du bassin versant surtout des plantations industrielles introduisant beaucoup d'engrais. Ces composés azotés favorisent la croissance des végétaux aquatiques et terrestres surtout des algues.

Les nitrates et les nitrites sont faiblement et positivement corrélés $(r=0,54)$. Ce qui signifie qu'ils évoluent ensemble dans le même sens. Les teneurs en composés azotés de la lagune Ono en dessous des normes de l'IBGE, (2005) n'auront pas une influence directe sur la pollution de cette lagune mais favorisent la croissance en masse des 
végétaux réduisant la transparence de la lagune.

Les fortes concentrations de l'ammonium observées dans la partie SudOuest au contact du fleuve Comoé s'expliqueraient par le fait que les eaux du fleuve Comoé qui se deversent dans la lagune Ono pendant la saison de crue sont chargées d'ammonium.

En milieux réducteurs, le nitrate se transforme en nitrite. En milieux fortement oxygénés l'ammonium se transforme intermédiairement en nitrite puis en nitrate qui constitue la phase finale. Il est donc à savoir qu'en présence d'oxygène, les nitrites sont instables et s'oxydent en nitrates. C'est ce qui explique la faible corrélation.

Aussi, les bactéries nitrifiantes décomposent la MO et libèrent l'ammoniac qui est soit utilisé par les plantes aquatiques, soit oxydé en nitrite.

Les teneurs élevées enregistrées en surface dans certaines zones de la lagune s'expliqueraient par le relargage des sédiments du fond vers la surface au moment des prélèvements.

La concentration moyenne de l'orthophosphate dans les eaux de la lagune Ono qui est de $0,693 \mathrm{mg} / \mathrm{l}$ est supérieure aux limites européennes comprises entre 0,005 $\mathrm{mg} / \mathrm{l}$ et $0,15 \mathrm{mg} / \mathrm{l}$. Cependant, en bassin on ne devrait pas dépasser $0,5 \mathrm{mg} / 1$ afin d'éviter la prolifération massive des algues (Ifremer, 2001). La bordure Nord de cette lagune favorise le développement des végétaux aquatiques et des algues suivant la variation de la teneur en orthophosphate dont leur décomposition induit l'eutrophisation de la lagune. Ces sels proviennent d'une part du lessivage des terres des plantations environnantes et des rejets des eaux usées domestiques et d'autre part de la décomposition de la matière organique. Comparée à la lagune Aghien-Potou, le fort prélèvement par les végétaux en lagune Ono réduit la teneur en ammonium $(0,033 \mathrm{mg} / \mathrm{l})$ et en nitrates $(0,977 \mathrm{mg} / \mathrm{l})$, mais avec des teneurs moyennes élevées en nitrites $(0,012 \mathrm{mg} / \mathrm{l})$. Les teneurs en lagune Aghien-Potou ont une moyenne de $23,04 \mathrm{mg} / \mathrm{l}$ en ions ammoniums, nitrates $4,26 \mathrm{mg} / \mathrm{l}$ et nitrites de $0,004 \mathrm{mg} / \mathrm{l}$ (Traoré et al., 2012).

La chlorophylle-a est un bon indicateur de la concentration de phytoplancton présent dans les eaux lagunaires qui est une des principales conséquences d'apports excessifs en sels d'azote et de phosphore dans les milieux aquatiques. La chlorophylle fait partie de la MO des eaux d'où leur bonne corrélation $(r=0,76)$. Selon l'IBGE, (2005), c'est un paramètre important de mesure du degré d'eutrophisation de l'eau. Les concentrations varient de $0,890 \mu \mathrm{g} / 1$ à $11,310 \mu \mathrm{g} / 1$ avec une

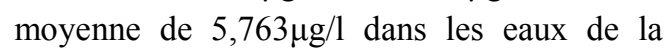
lagune Ono. Pellerin et al., (2011) qualifient une eau de concentration comprise entre 9-25 $\mu \mathrm{g} / \mathrm{l}$ de chlorophylle-a et une transparence comprise entre 1-2 m, d'eau eutrophe. Ainsi, la lagune Ono est eutrophe dans le secteur de Binsérémé à proximité des plantations d'ananas et mésotrophe à oligotrophe en remontant vers le Nord-Est. Selon, les normes françaises et comme indiqué par Abdellaoui et al., (2005), ces eaux présentent une dégradation par eutrophisation.

La teneur en chlorophylle-a dépassant $10 \mu \mathrm{g} / \mathrm{l}$, caractérisant un état très eutrophisé comme observé dans la lagune de Canet par Ifremer, (2013). Rappelons que la concentration en chlorophylle-a permet de mettre en évidence la productivité phytoplanctonique d'une eau (Ifremer, 2002).

Ifremer, (2002) a établi cinq classes de productivité : de 0 à $5 \mu \mathrm{g} / \mathrm{l}$ faible, de 5 à 10 $\mu \mathrm{g} / 1$ moyenne, de 10 à $20 \mu \mathrm{g} / \mathrm{l}$ forte, et $>20$ $\mu \mathrm{g} / \mathrm{l}$ très forte. La lagune Ono présente donc une moyenne productivité depuis l'extrémité Sud en passant par la bordure Nord jusqu'au village Ono. Une zone de forte productivité vers le village de Binsérémé indiquant la zone de rejet des eaux des plantations environnantes.

\section{Conclusion}

Cette étude axée sur l'analyse du comportement des paramètres 
d'eutrophisation de la lagune Ono a permis d'examiner l'état trophique de cette eau pendant la crue du fleuve Comoé. La réaction de ce système lagunaire face à l'influence des rejets agricoles, industriels et domestiques est perçue dans la dynamique des paramètres étudiés. La matière organique et la transparence évoluent suivant les mêmes séquences de variation aboutissant à une pollution organique. Par ailleurs les teneurs en sel d'azote et d'orthophosphate sont à la base $\mathrm{du}$ « bloom » phytoplanctonique réduisant la surface de cette lagune. Elles augmentent la concentration de la chlorophylle-a contribuant à réduire la transparence. L'orthophosphate avec des teneurs élevées constitue le facteur limitant de l'évolution végétative de cette lagune. La corrélation inverse entre l'oxygène dissous et la matière organique relève de la forte croissance des végétaux et de leur décomposition demandant de l'oxygène. La lagune Ono est d'un état eutrophe à oligotrophe du Sud-Ouest vers le Nord-Est. Hormis les milieux aquatiques frontaliers, l'hydrologie de la lagune Ono est contrôlée par les influences anthropiques. Une alternative est de réduire en amont l'apport des éléments nutritifs dans cette lagune.

\section{CONFLIT D'INTERETS}

Les auteurs déclarent qu'il n'y a aucun conflit d'intérêts sur cet article.

\section{CONTRIBUTIONS DES AUTEURS}

Les auteurs de cet article ont contribué aux différents travaux et à la rédaction du manuscrit.

\section{REMERCIEMENTS}

Nous exprimons nos sincères remerciements au Centre Ivoirien Antipollution (CIAPOL) et le Laboratoire de Géologie Marie et Sédimentologie (GEOMARSE) de l'Université Félix Houphouët Boigny de Cocody Abidjan.

\section{REFERENCES}

Ayah M, Grybos M, Tampo L, Bawa LM. 2015. Qualité et pollution des eaux d'un Hydrosystème littoral tropical : cas du système lagunaire de Lomé, Togo. European Scientific Journal édition, 11(15) : 25p. DOI : htt://eujournal.org/index.php/esj/issue/vi ew/164

Abdellaoui B, Najih M, Idhalla M. 2005. Fonctionnement hydrologique et risque d'eutrophisation de la lagune de Nador. Rapport final LagMar 10p. https://www.academia.edu/12717331

Coulibaly AS, Touré M, Diangoné E, Sylvain M, Aka K, Gérard B. 2014b. Impacts des rejets accidentels sur la qualité environnementale des sédiments de la rade portuaire d'Abidjan (lagune Ebrié ; Côte d'Ivoire). Int. J. Biol. Chem. Sci., 8(6): 2842-2848. DOI: http://dx.doi.org/10.4314/ijbcs.v8i6.40

Eyi AJ, Konan KJ, Tano K, N'da K, Atsé BC. 2016. Étude préliminaire des communautés itchyofauniques de la lagune Ono (Côte d'Ivoire). Journal of Applied Biosciences, 104: 9894 - 9903. DOI: http://dx.doi.org/10.4314/jab.v104i1.1

IBGE. 2005. Qualité physico- chimique et chimique des eaux de surface : cadre général. Les données de l'IBGE : "L'eau à Bruxelles" 16p. Document. Environnement.

Brussels/opac_css/elecfile/Eau \% 202

Ifremer. 2001. Suivi des paramètres trophiques dans l'eau des étangs de la Narbonnaise.

$52 \mathrm{p}$. https://archimer.ifremer.fr

Pellerin H, Dorioz JM, Morel C. 2011. Bilan environnemental du phosphore. In "Sols et environnements" pp. 358-377. Dunod, Paris.

Rapport Ifremer. 2002. Evaluation de l'état d'eutrophisation des eaux côtières et estuariennes de Basse-Normandie 78 p. 
https://archimer.ifremer.fr/doc/00087/19 $781 / 17423$.

Rapport Ifremer. 2013. Analyse statistique des données du RSL. Etude des trajectoires écologiques des lagunes entre 2001 et 2012. RST-LER/LR 13-06. https://archimer.ifremer.fr/doc/00173/28 $423 /$

SEEE. 2008. Fiche sur le nouveau système d'évaluation de la qualité des eaux, Maroc, $5 \mathrm{p}$.

https://journals.openedition.org/mediterr anee/6221

Traoré A, Soro G, Kouadio KE, Bamba SB, Oga SM, Soro N, Biemi J. 2012. Evaluation des paramètres physiques, chimiques et bactériologiques des eaux d'une lagune tropicale en période d'étiage : la lagune Aghien (Côte d'Ivoire). Int. J. Biol. Chem. Sci., 6(6): 7048-7058.

DOI: http://dx.doi.org/10.4314/ijbcs.v6i6.40.

Tohouri P, Adja GM, Soro G, Aké EG, Konan IN, Biemi J. 2017. Qualité physico- chimique en saison pluvieuse des eaux de surface de la région de Bonoua (sudest de la Côte d'Ivoire). International Journal of Innovation and Applied Studies, 20: 28-41. DOI: http://www.ijias.issr-journals.org.

Wognin AV, N'guessan YM, Assalé FJP, Aka AM, Ccoulibaly AS, Mondé S, Aka K. 2017. Les éléments traces métalliques dans la lagune Ebrié : distribution saisonnière, niveau de contamination et qualité environnementale des sédiments. Int. J. Biol. Chem. Sci., 11(2): 911-923. DOI: http://dx.doi.org/10.4314/ijbcs.v11i2.30

Yao KM, Métongo BS, Trokourey A, Bokra Y. 2009. La pollution des eaux de la zone urbaine d'une lagune tropicale par les matières oxydables (lagune Ébrié, Côte d'Ivoire). International Journal of Biological and Chemical Sciences, 3(4): 755-770.

DOI: htt://dx.doi.org/ijbcs.v3i4.47168. 